\title{
Toxic Anterior Segment Syndrome following a Triple Descemet's Stripping Automated Endothelial Keratoplasty Procedure
}

\author{
Nir Sorkin David Varssano \\ Department of Ophthalmology, Tel Aviv Medical Center and the Sackler Faculty \\ of Medicine, Tel Aviv University, Tel Aviv, Israel
}

\section{Key Words}

Toxic anterior segment syndrome · Triple procedure - Descemet's stripping automated endothelial keratoplasty

\begin{abstract}
Purpose: To present a unique case of a 58-year-old female with toxic anterior segment syndrome (TASS), following a triple procedure: Descemet's stripping automated endothelial keratoplasty (DSAEK), phacoemulsification and posterior chamber intraocular lens implantation.

Methods: The patient was treated with topical dexamethasone sodium phosphate $0.1 \%$ and topical atropine sulfate $1 \%$. Due to a slow improvement in her clinical status, oral prednisone $1 \mathrm{mg} / \mathrm{kg} /$ day was added.

Results: The anterior chamber reaction improved gradually, with tapering down of topical and oral treatment, until a complete resolution of the anterior chamber reaction was observed.

Conclusions: Taking into account the estimated volume of DSAEK triple procedures performed worldwide, we would expect an annual incidence of several TASS cases, following triple DSAEK procedures. However, we were unable to find any such previous reports in the literature. This fact raises questions regarding the cause of reduced TASS incidence following triple DSAEK procedures.
\end{abstract}




\section{Introduction}

Toxic anterior segment syndrome (TASS) is an acute, sterile anterior chamber inflammation, which may develop following anterior segment surgery [1]. Most cases are reported after uneventful cataract surgery.

In patients requiring both cataract extraction and endothelial keratoplasty, a Descemet's stripping automated endothelial keratoplasty (DSAEK) triple procedure, consisting of phacoemulsification and intraocular lens (IOL) implantation, followed by DSAEK, may be the procedure of choice [2].

Up to the present, only two reports of keratoplasty-related TASS have been published, both following penetrating keratoplasty. The first was a case series of 8 patients with TASS-like, noninfectious, severe, early inflammation [3]. The second, reported a large outbreak of TASS (24 cases out of 94 procedures performed), presenting as postoperative keratitis with inflammatory anterior chamber reaction mainly hypopion and cells. This outbreak was found to be caused by faulty sterilization of the guided trephine system [4]. To our knowledge, no reports of TASS following DSAEK or a triple procedure have been published. Herein is presented a case of TASS following a triple procedure: DSAEK, phacoemulsification and posterior chamber IOL implantation.

\section{Case Report}

A 58-year-old female, suffering from bilateral Fuchs' endothelial dystrophy, bilateral cataract and corneal subepithelial scarring in her left cornea, following an adenovirus infection, underwent an uneventful DSAEK triple procedure in her left eye: phacoemulsification, implantation of a posterior chamber IOL and DSAEK. The follow-up examination, one day postoperatively, was unremarkable. Intraocular pressure was $13 \mathrm{~mm} \mathrm{Hg}$, best-corrected visual acuity was 20/200, and the graft was attached to the recipient cornea, with a deep anterior chamber. Treatment with topical dexamethasone sodium phosphate $0.1 \%$ and ofloxacin $0.3 \%$ administered four times daily was initiated.

Five days following the surgery, the patient presented with decreased vision in her left eye, which had begun 24 h postoperatively. On examination of her left eye, the best-corrected visual acuity was 20/720. Intraocular pressure was $11 \mathrm{~mm} \mathrm{Hg}$. Slit-lamp examination (fig. 1) revealed conjunctival hyperemia, a completely attached and centrally located endothelial corneal graft, deep anterior chamber with a cellular reaction of $3+$ and prominent flare measuring $3+$. The pupil contained multiple fibrin fibers, with posterior synechiae spanning 360 degrees. The posterior chamber IOL was properly positioned. Visualization of the posterior segment was poor due to the clinical status of the anterior segment - no details could be observed, but for a red light reflex.

The patient was started on hourly topical dexamethasone sodium phosphate $0.1 \%$. Due to the extensive posterior synechiae, the patient received a short and intensive course of topical tropicamide $0.5 \%$ and phenylephrine hydrochloride $10 \%$, followed by topical atropine sulfate $1 \%$. Follow-up examination, one day after the initiation of treatment, revealed significant improvement in the anterior chamber cellular reaction with no evident cells or flare, and residual posterior synechiae spanning 60 degrees. The posterior segment could be well visualized, showing no inflammatory involvement of the retina and vitreous. Fibrin fiber accumulation was only mildly improved and therefore, the patient was started on oral prednisone $1 \mathrm{mg} / \mathrm{kg} /$ day. Due to the early onset of visual deterioration ( $24 \mathrm{~h}$ postoperatively), the normal appearing posterior segment (visualized shortly after initiation of treatment) and the rapid clinical response to the above treatment, a clinical diagnosis of infectious endophthalmitis was ruled out. We therefore decided not to perform a vitreal diagnostic (or interventional) procedure. Follow-up examinations on days 3, 8 and 10 following 
initiation of treatment, revealed gradual absorption of fibrin and no evident posterior synechiae. Oral and topical steroids were gradually tapered down.

At follow-up examination 8 weeks following the TASS diagnosis, the patient's best-corrected visual acuity was $20 / 50$, with a spherical correction of +1.25 diopter and a cylindrical correction of -1.75 diopter $\left(170^{\circ}\right.$ axis $)$. Intraocular pressure was $14 \mathrm{~mm} \mathrm{Hg}$, with a clear, attached, thin endothelial corneal graft. Epithelial corneal edema was observed only outside the margins of the endothelial graft. No recipient corneal edema was observed inside the margins of the endothelial corneal graft. No evidence of an anterior chamber reaction, fibrin formation or posterior synechiae was observed.

\section{Discussion}

According to the Eye Bank of America Association, 42,606 corneal transplants were performed in the USA in 2009, out of which 18,221 were endothelial keratoplasty procedures ('2009 Eye Banking Statistical Report', Washington, DC, Eye Bank Association of America). The World Health Organization (WHO) estimates that about 120,000 corneal transplants are performed worldwide each year ('Human Organ and Tissue Transplantation - Report by the Secretariat', submitted at the 112th session of the World Health Organization Executive Board, May 2003). There is no sufficient data in the literature regarding the volume of triple DSAEK procedure surgeries performed worldwide. We estimate they accumulate to several thousand performed annually.

The frequency of TASS reported in the literature is about $0.25-0.8 \%[5,6]$. Therefore, we would expect an annual incidence of several TASS cases, following triple DSAEK procedures. However, we were unable to find any such previous reports in the literature. It is probable that the cataract extraction constituent of the triple DSAEK procedure is responsible for the occurrence of TASS in our patient. Nevertheless, the fact that there is no previous report of TASS following such a procedure raises questions regarding the cause of the reduced incidence of TASS following triple DSAEK procedures.

Due to the fact that the inflammatory reaction may jeopardize the integrity of the endothelial graft and reduce the chances of a successful transplant [7], we intend to closely monitor this patient and apply a more gradual tapering down of her current treatment.

\section{Disclosure Statement}

No conflicting relationship exists for any author. 


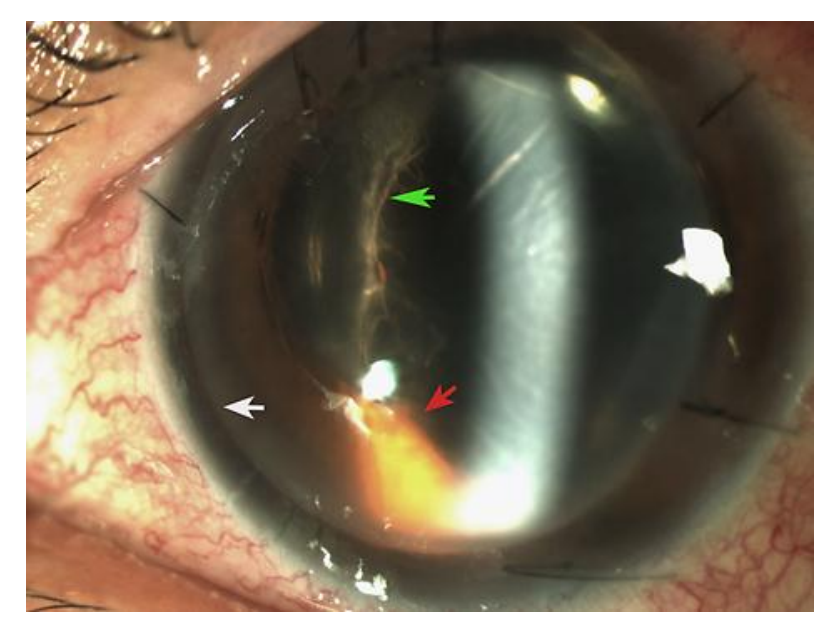

Fig. 1. Slit-lamp photograph of left eye showing the endothelial graft border (white arrow), posterior synechia (red arrow) and fibrin fibers (green arrow).

\section{References}

1 Mamalis N: Toxic anterior segment syndrome (editorial). J Cataract Refract Surg 2006;32:181-182.

$>2$ Inoue Y: Corneal triple procedure. Semin Ophthalmol 2001;16:113-118.

-3 Lim SJ, Kim SY, Kim MS: Noninfectious severe early chamber reaction after penetrating keratoplasty. J Korean Ophthalmol Soc 2007;48:343-347.

4 Maier P, Birnbaum F, Böhringer D, Reinhard T: Toxic anterior segment syndrome following penetrating keratoplasty. Arch Ophthalmol 2008;126:1677-1681.

5 Cutler Peck CM, Brubaker J, Clousers S, Danford C, Edelhauser HE, Mamalis N: Toxic anterior segment syndrome: common causes. J Cataract Refract Surg 2008;36:1067-1080.

6 Ozcelik ND, Eltutar K, Bilgin B: Toxic anterior segment syndrome after uncomplicated cataract surgery. Eur J Ophthalmol 2010;20:106-114.

-7 Dua HS, Azuara-Blanco A: Corneal allograft rejection: risk factors, diagnosis, prevention, and treatment. Indian J Ophthalmol 1999;47:3-9. 